\title{
Could recombinant insulin compounds contribute to adenocarcinoma progression by stimulating local angiogenesis?
}

\author{
K. L. Rensing • F. M. Houttuijn Bloemendaal • E. M. Weijers • D. J. Richel • \\ H. R. Büller • P. Koolwijk • C. M. van der Loos • Th. B. Twickler • \\ J. H. von der Thüsen
}

Received: 16 December 2009 / Accepted: 19 January 2010/Published online: 25 February 2010

(C) The Author(s) 2010. This article is published with open access at Springerlink.com

\begin{abstract}
Aims/hypothesis Negative effects on the progression of adenocarcinomas by hyperinsulinaemia and the insulin analogue glargine (A21Gly,B31Arg,B32Arg human insulin) have recently been suggested. Most actions of this insulin analogue have hitherto been explained by direct stimulation of growth potential of neoplastic cells and by its IGF-1 related properties. However, insulinstimulated angiogenesis could be an additional factor involved in tumour progression and clinical outcomes associated with cancer.
\end{abstract}

K. L. Rensing and F. M. Houttuijn Bloemendaal contributed equally to the present study.

K. L. Rensing $(\bowtie) \cdot$ F. M. Houttuijn Bloemendaal $•$ H. R. Büller •

Th. B. Twickler

Department of Vascular Medicine,

Academic Medical Center/University of Amsterdam,

Meibergdreef 9 ,

1105 AZ Amsterdam, the Netherlands

e-mail: k.l.rensing@amc.uva.nl

E. M. Weijers $\cdot$ P. Koolwijk

Institute for Cardiovascular Research, Laboratory for Physiology,

VU University Medical Center,

Amsterdam, the Netherlands

D. J. Richel

Department of Internal Medicine,

Academic Medical Center/University of Amsterdam,

Amsterdam, the Netherlands

C. M. van der Loos $\cdot$ J. H. von der Thüsen

Department of Pathology,

Academic Medical Center/University of Amsterdam,

Amsterdam, the Netherlands
Methods Five types of human adenocarcinoma (breast, colon, pancreas, lung and kidney) were evaluated for the presence of insulin receptors (IRs) on angiogenic structures. In an in vitro angiogenesis assay, various commercially available insulin compounds were evaluated for their potential to increase capillary-like tube formation of human microvascular endothelial cells (hMVEC). Insulin compounds used were: human insulin, insulin lispro (B28Lys, B29Pro human insulin), insulin glargine and insulin detemir (B29Lys[e-tetradecanoyl],desB30 human insulin).

Results Insulin receptors were found to be strongly expressed on the endothelium of microvessels in all evaluated adenocarcinomas, in addition to variable expression on tumour cells. Low or no detectable expression of IRs was seen on microvessels in extratumoral stroma. Incubation with commercially available insulin compounds increased capillary-like tube formation of hMVEC in vitro.

Conclusions/interpretation Our results suggest that all tested insulin compounds may stimulate tumour growth by enhancing local angiogenesis. Future studies need to confirm the association between insulin therapy in type 2 diabetes and tumour progression.

Keywords Angiogenesis · Insulin · Tumour progression · Type 2 diabetes

$\begin{array}{ll}\text { Abbreviations } \\ \text { 3D } & \text { Three-dimensional } \\ \text { bFGF } & \text { Basic fibroblast growth factor } \\ \text { hMVEC } & \text { Human microvascular endothelial cells } \\ \text { IGF-1R } & \text { IGF-1 receptor } \\ \text { IR } & \text { Insulin receptor }\end{array}$




\section{Introduction}

Recent retrospective studies suggest that patients with type 2 diabetes who use the insulin analogue insulin glargine (A21Gly,B31Arg,B32Arg human insulin) might have an increased risk of developing cancer. Hemkens et al. found a positive association between daily insulin dosage and the incidence of malignant neoplasms. Interestingly, this association was present for human insulin and insulin analogues such as glargine, lispro (B28Lys,B29Pro human insulin) and aspart (B28Asp human insulin). Moreover, after adjusting for insulin dose, they found a dose-dependently increased risk of cancer in patients treated with insulin glargine compared with patients treated with human insulin with an adjusted hazard ratio of 1.31 (95\% CI 1.20-1.42) for a daily dose of 50 IU [1]. In another report, Jonasson et al. mentioned that patients taking insulin glargine had an almost twofold increased risk of breast cancer compared with patients using other types of insulin [2]. The SDRN Epidemiology Group observed a similarly increased risk, but attributed this to an allocation bias [3]. A study by Currie et al. showed no association between insulin analogues and cancer progression as compared with human insulin, but did show that patients using any kind of insulin had a higher risk of developing adenocarcinomas than those on metformin [4].

An important point to elucidate in this context is the pathophysiological mechanism through which insulin could be involved in tumour progression. In their editorial accompanying the above-mentioned articles, Smith and Gale [5] noted that it is surprising that these studies were able to detect differences in cancer rates after only a few years of insulin exposure, because cancer takes many years to develop. Supported by existing literature, they suggest that, if insulin does indeed influence cancer incidence, this is possibly due to stimulatory effects on pre-existing malignant foci rather than to de-novo carcinogenesis. Insulin is known to augment mitogenesis and proliferation of distinctive cell types and its effect can be mediated through insulin receptors (IRs), insulin like growth factor-1 (IGF-1) receptors (IGF-1Rs) or hybrid IR-IGF-1Rs. IRs and IGF-1Rs are frequently present on pre-existing tumour cells and this could give insulin a molecular pathway to contribute to tumour expansion [5]. However, we recently showed that IRs are also abundantly present on angiogenic structures in human atherosclerotic plaques suggesting a biological role of insulin on local angiogenesis [6]. Furthermore, it has been shown that insulin is involved in angiogenesis during wound healing [7]. We therefore hypothesised that such a mechanism might also be involved in tumour expansion and tumour growth.

We analysed whether IRs are expressed on angiogenic structures in adenocarcinomas. In addition, we evaluated the effect of commercially available human insulin and insulin analogues on capillary-like tube formation of human microvascular endothelial cells (hMVEC) in vitro.

\section{Methods}

Collection of specimens Tissue was obtained from archival material of resection specimens in accordance with guidelines set out by the 'Code for Proper Secondary Use of Human Tissue' of the Dutch Federation of Biomedical Scientific Societies. Included were adenocarcinomas from breast $(n=15)$, colon $(n=15)$, pancreas $(n=15)$, lung $(n=15)$ and kidney $(n=15)$. As a control, we investigated peritumoural parenchyma of the included specimens, especially of those known to be insulin-sensitive (kidney and pancreas).

Immunohistochemical analysis All specimens were routinely fixed in buffered formalin and embedded in paraffin. After heat-induced epitope retrieval in Tris-EDTA pH9.0 $\left(20 \mathrm{~min}\right.$ at $\left.98^{\circ} \mathrm{C}\right)$, anti-IR $\beta$-subunit antibody $(1 \mu \mathrm{g} / \mathrm{ml}$, $60 \mathrm{~min}$, room temperature; Millipore/Chemicon, Temecula, CA, USA) and anti-IGF1-R $\alpha$-subunit antibody $(0.5 \mu \mathrm{g} / \mathrm{ml}$, $60 \mathrm{~min}$, room temperature; Millipore/Chemicon) were immunostained using a three-step polymer detection system (ImmunoLogic, Duiven, the Netherlands). Horseradishperoxidase activity was visualised in brown with diaminobenzidine (DAB+; Dako, Glostrup, Denmark). Negative controls consisted of concentration-matched isotype controls (Dako).

Expression of IRs on endothelial and epithelial cells within adenocarcinoma and in surrounding tissue was assessed using a semi-quantitative score: $0=$ no IR expression, $1=$ minimal IR expression, $2=$ moderate IR expression, $3=$ strong IR expression.

Immuno double-staining combining anti-IR antibody in red (Vector Red; Vector Laboratories, Burlingame, CA, USA) and anti-CD31 (Dako) antibody in blue (Vector Blue) was performed with the sequential double alkaline phosphatase method [8].

Spectral imaging Analysis of double staining slides was performed with spectral imaging using a multispectral imaging system (Nuance VIS-FL; Cambridge Research Instrumentation, Woburn, MA, USA). Data sets were acquired from $420-72 \mathrm{~nm}$ at $20 \mathrm{~nm}$ intervals. Spectral library of single-Vector Red and single-Vector Blue was used to unmix the double staining into the individual components. Using Nuance software version 2.8 (Cambridge Research Instrumentation), an exclusive image of IR/CD31 co-localisation as well as fluorescent-like pseudocoloured images were created [9]. 
In vitro angiogenesis Individual human foreskin MVEC (hMVEC), obtained from three different donors, were confluently seeded in M199 supplemented with $10 \%$ (vol./vol.) heat-inactivated human serum and 10\% (vol./ vol.) newborn calf serum on a three-dimensional fibrin matrix, prepared with $2 \mathrm{mg} / \mathrm{ml}$ fibrinogen (Calbiochem, La Jolla, CA, USA) and 0.1 U/ml thrombin (Organon, Boxtel, the Netherlands), as previously described [10]. To study the effect of different insulin compounds and IGF-1 on capillary-like tube formation, hMVEC were stimulated with $10 \mathrm{ng} / \mathrm{ml}$ basic fibroblast growth factor (bFGF) (Peprotech, London, UK) and $10 \mathrm{ng} / \mathrm{ml} \mathrm{TNF-} \alpha$ (Sigma Aldrich, St Louis, MO, USA) in absence or presence of $1 \times 10^{-9}, 1 \times$ $10^{-8}$ or $1 \times 10^{-6} \mathrm{~mol} / 1$ of human insulin (actrapid), shortacting insulin analogue (insulin lispro), long-acting insulin analogues (insulin detemir [B29Lys(e-tetradecanoyl), desB30 human insulin], insulin glargine) or IGF-1 (GroPep, Adelaide, Australia) in triplicate or quadruplicate. Medium was refreshed every other day. After 7 to 12 days of incubation, the formation of tubular structures was analysed by phase contrast microscopy and quantified with Optimas image analysis software (Media Cybernetics, Bethesda, MD, USA). The use of hMVEC was approved by the ethical committee of the VU University Medical Centre, Amsterdam, the Netherlands.

Statistical analysis Semi-quantitative expression data were compared by means of the Wilcoxon matched-pairs signedranks test. Results from the in vitro angiogenesis experiments were combined and are presented as mean \pm SEM. Statistical differences were determined by a paired $t$ test, using Prism 4 (GraphPad, San Diego, CA, USA). $p<0.05$ was considered to be statistically significant.

\section{Results}

Presence of insulin receptors (immunohistochemical evaluation) The endothelium of microvessels in the tumour
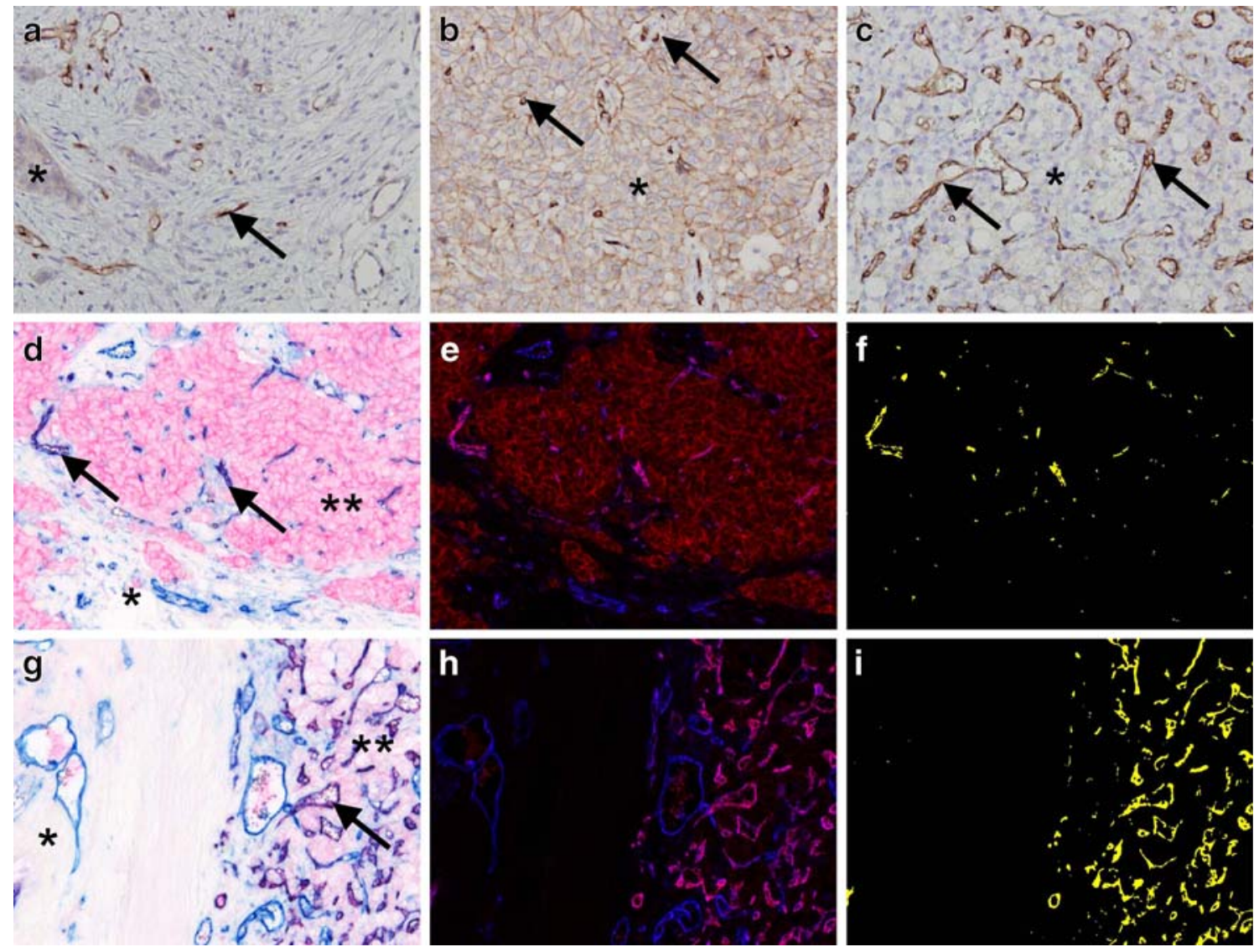

Fig. 1 Immunohistochemical staining for the insulin receptor in colon (a), breast (b) and renal (c) adenocarcinoma (magnification $\times 400$ ). Tumour cells are variably positive $(*)$, whereas neovessels in the tumour stroma are invariably strongly positive (arrows). Doublestaining (magnification $\times 200$ ) demonstrates colocalisation (purple, arrows) for the IR (red) and CD31 (blue) in neovessels in the tumour stroma in breast (d) and kidney carcinoma (g), which was confirmed by spectral imaging analysis for breast $(\mathbf{e}, \mathbf{f})$ and kidney $(\mathbf{h}, \mathbf{i})$ carcinoma respectively, with colocalisation in magenta/yellow. d, g Endothelial cells in the surrounding non-tumour stroma show no or very little IR staining $(*)$ compared with endothelial cells in the tumour stroma $(* *)$ 
Fig. 2 Mean score of semiquantitative insulin receptor expression on (a) endothelial cells and (b) epithelial cells in tumour (T) vs surrounding parenchyma (P). Scores: $0=$ no, $1=$ minimal, $2=$ moderate, $3=$ strong IR expression. $n=15$ for each tumour type; $* * p<0.01$, $* * * p<0.001$ and ${ }^{\dagger} p=0.02$ compared with $\mathrm{T}$
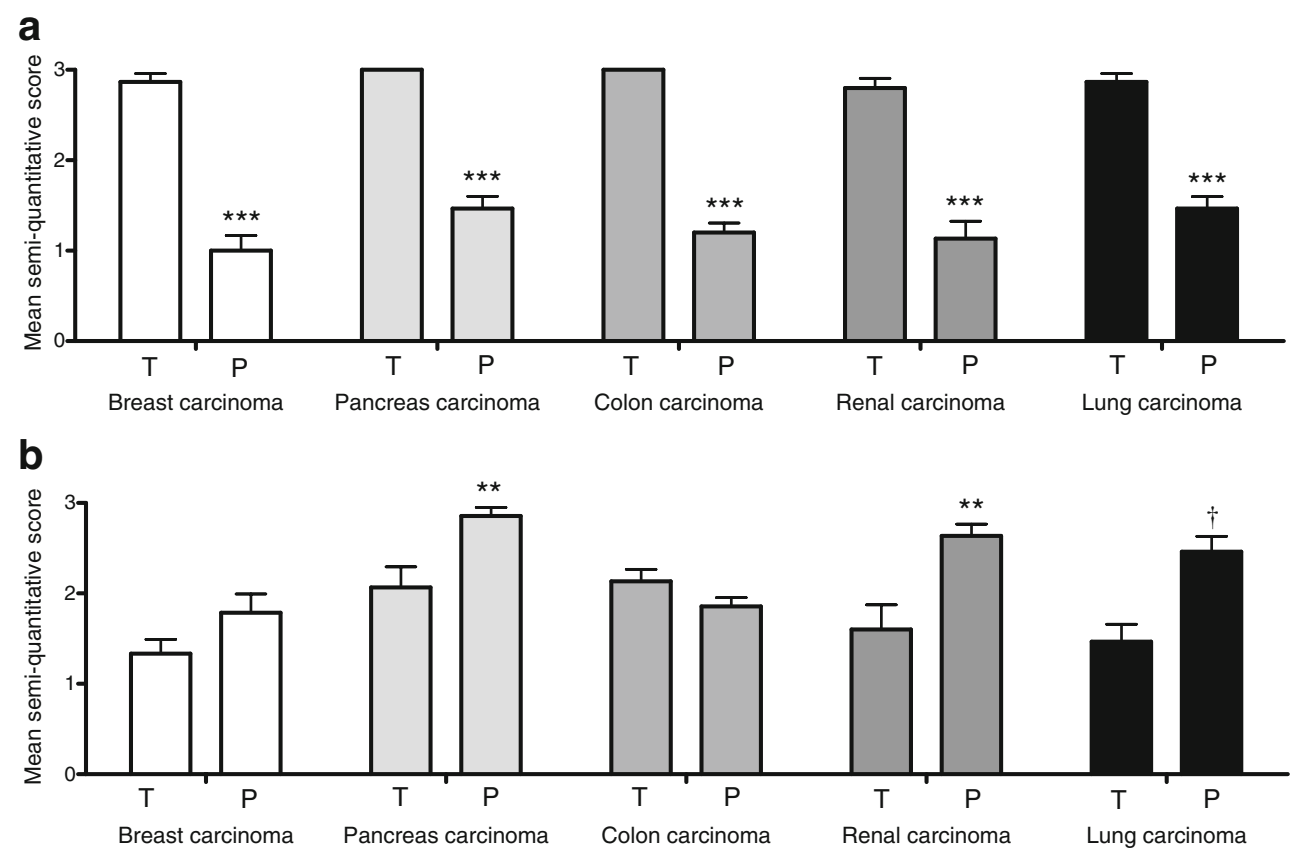

stroma of all examined tumour types was consistently and strongly positive for the IR, with much reduced or absent staining in the microvasculature of surrounding non-tumour stroma and parenchymal tissue, including that of insulinsensitive tissues (kidney and pancreas; Fig. 1). The endothelial nature of this staining pattern was confirmed by double immunohistochemistry for the IR and CD31, aided by spectral imaging analysis. In addition, all tumour types showed variably cytoplasmic and/or membranous staining on epithelial cells for the IR, ranging from none to strong expression.

Staining intensity was scored semi-quantitatively, showing a clear significant increased staining in tumour microvessels as compared with microvessels in non-tumour stroma for all tumour types. The pattern for epithelial staining was more complex and revealed more intensive staining in the parenchyma for most tumour types compared with the neoplastic epithelium (Fig. 2). Staining for IGF-1R was weak on endothelial cells, with no differences between tumour and non-tumour tissue (data not shown).

\section{Capillary-like tube formation (in vitro experiments) Figure 3} shows the combined results of five to eight in vitro experiments, in which the effect of insulin compounds on capillary-like tube formation of hMVEC in 3D fibrin matrices was assessed. Compared with stimulation with bFGF and TNF- $\alpha$ alone (control), addition of distinctive insulin compounds $\left(1 \times 10^{-9}, 1 \times 10^{-8}\right.$ and $\left.1 \times 10^{-6} \mathrm{~mol} / \mathrm{l}\right)$ to the incubation medium led to significant increased capillary-like tube formation of hMVEC. The extent of capillary-like tube formation varied between compounds, ranging from a 1.6- to 2.3-fold increase compared with control. Stimulation with IGF-1 $\left(1 \times 10^{-9}, 1 \times 10^{-8}\right.$ and $1 \times$ $\left.10^{-6} \mathrm{~mol} / \mathrm{l}\right)$ gave a similar increase in capillary-like tube formation (data not shown).

\section{Discussion}

This study suggests that distinctive insulin compounds (exceeding a certain daily dose) could contribute to tumour growth by enhancing local angiogenesis, possibly parallel to the established direct mitogenic and proliferative effects of insulin on tumours [5].

Our observations showed that common adenocarcinomas, which frequently afflict patients with type 2 diabetes, contain large numbers of neovessels and that these vessels

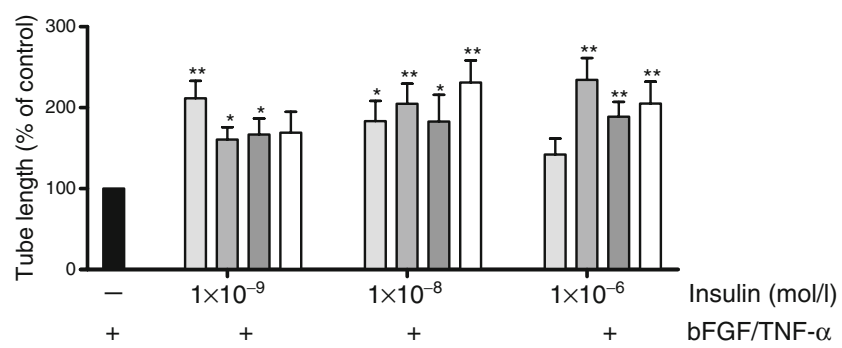

Fig. 3 Increased capillary-like tube formation by hMVEC after stimulation with different insulin compounds. Black bar, control; light grey bars, human insulin; mid-grey bars, insulin lispro; dark grey bars, insulin detemir; white bars, insulin glargine. All human insulin compounds enhanced, to varying degrees, capillary-like tube formation compared with stimulation with $\mathrm{bFGF} / \mathrm{TNF}-\alpha$ alone. Each experiment was done in triplicate or quadruplicate. $n=5$ for $1 \times 10^{-9}$ $\mathrm{mol} / \mathrm{l}, n=8$ for $1 \times 10^{-8} \mathrm{~mol} / 1$ and $1 \times 10^{-6} \mathrm{~mol} / 1$ insulin; $* p<0.05$ and $* * p<0.01$ compared with control 
abundantly express IRs, independent of tumour type. This indicates that local expression of IR on neovessels is a rather general phenomenon in the process of tumour angiogenesis and that high volumes of exogenous insulin could have side effects. Stimulation of these receptors with insulin could promote angiogenesis and subsequent tumour progression. It should be noted that tumours can have high expression of hybrid receptors and particularly of the IR-A isotype, which is not readily detectable with currently available antibodies. Therefore, the IR expression detected may be an underestimate.

The effect of insulin on angiogenesis has been observed before. Liu et al. [7] recently reported that insulin stimulates hMVEC migration and two-dimensional Matrigel tube formation exclusively through the IR. Our in vitro experiments showed that not only the long-acting insulin analogue glargine enhances capillary-like tube formation, but also all other evaluated insulin compounds. Hence, insulin glargine is not an exclusive candidate for an assumed relationship between increased tumour growth and insulin use in patients with type 2 diabetes.

It is likely that the added insulin at high concentrations may act via both IRs and IGF-1Rs. This might be the case for insulin concentrations of $1 \times 10^{-6}$ and $1 \times 10^{-8}$, but not for $1 \times 10^{-9} \mathrm{~mol} / \mathrm{l}$. Moreover, differences in capillary-like tube formation between insulin detemir and insulin glargine were not found. It is known that insulin glargine has a much higher affinity for the IGF-1R compared with insulin detemir [11]. Therefore, involvement of the IGF-1R becomes less probable. Furthermore, since we found increased IR expression and no increased IGF-1R expression on microvessels within adenocarcinomas, we assume that in vivo the insulin $\times$ IR interactions are likely to be of paramount importance for insulin-mediated angiogenic effects in adenocarcinomas.

We would have preferred to use human tumour-derived endothelial cells, instead of foreskin-derived hMVEC for our in vitro experiments. However, to our knowledge, no published reports describe the isolation and long-term culturing of endothelial cells from human tumour tissue. Since endothelial cells within tumour tissue are of benign origin and adenocarcinomas normally contain much fibrin, we consider our in vitro angiogenesis assay, which looks at tube formation of benign hMVEC into a three-dimensional fibrin matrix, to be appropriate.

In short, the current results suggest that insulin and insulin analogues play a biological role in tumour growth by stimulating local IRs with a subsequent increase in angiogenesis. Without any doubt, additional prospective studies will be required in order to confirm a possible relationship between insulin therapy and tumour progression either by direct effects of insulin on tumour growth or stimulation of local angiogenesis.

Acknowledgements Th. B. Twickler received a grant from the Netherlands Organization of Science (NWO/ZonMW). J. H. von der Thüsen is an awardee of a Dutch Heart Foundation Dekker grant (NHS 2008 T050) and P. Koolwijk received a grant from the Dutch Program of Tissue Engineering (STW/DPTE, grant number BGT.6733).

Duality of interest The authors declare that there is no duality of interest associated with this manuscript.

Open Access This article is distributed under the terms of the Creative Commons Attribution Noncommercial License which permits any noncommercial use, distribution, and reproduction in any medium, provided the original author(s) and source are credited.

\section{Reference}

1. Hemkens LG, Grouven U, Bender R et al (2009) Risk of malignancies in patients with diabetes treated with human insulin or insulin analogues: a cohort study. Diabetologia 52:1732-1744

2. Jonasson JM, Ljung R, Talback M, Haglund B, Gudbjornsdottir S, Steineck G (2009) Insulin glargine use and short-term incidence of malignancies - a population-based follow-up study in Sweden. Diabetologia 52:1745-1754

3. Colhoun HM, SDRN Epidemiology Group (2009) Use of insulin glargine and cancer incidence in Scotland: a study from the Scottish Diabetes Research Network Epidemiology Group. Diabetologia 52:1755-1765, Erratum 32: 2470

4. Currie CJ, Poole CD, Gale EA (2009) The influence of glucoselowering therapies on cancer risk in type 2 diabetes. Diabetologia 52:1766-1777

5. Smith U, Gale EA (2009) Does diabetes therapy influence the risk of cancer? Diabetologia 52:1699-1708

6. Rensing KL, Houttuijn Bloemendaal F, Weijers EM et al (2009) Potential role for insulin in sprouting of neovessels within atherosclerotic plaques; New pathways of microvascular complications in macrovascular disease of diabetes. Atherosclerosis Supplement 10:1090, Abstract

7. Liu Y, Petreaca M, Martins-Green M (2008) Cell and molecular mechanisms of insulin-induced angiogenesis. J Cell Mol Med 12:1-13

8. Van der Loos CM, Teeling P (2008) A generally applicable sequential alkaline phosphatase immunohistochemical double staining. J Histotechnol 31:119-127

9. Van der Loos CM (2008) Multiple immunoenzyme staining: methods and visualizations for the observation with spectral imaging. J Histochem Cytochem 56:313-328

10. Koolwijk P, van Erck MG, de Vree WJ et al (1996) Cooperative effect of TNFalpha, bFGF, and VEGF on the formation of tubular structures of human microvascular endothelial cells in a fibrin matrix. Role of urokinase activity. J Cell Biol 132:1177-1188

11. Kurtzhals P, Schaffer L, Sorensen A et al (2000) Correlations of receptor binding and metabolic and mitogenic potencies of insulin analogs designed for clinical use. Diabetes 49:999-1005 